$$
\begin{aligned}
& \text { قياس الرهاب الاجتماعي لاى طالبات الصف الخامس اعدادي }
\end{aligned}
$$

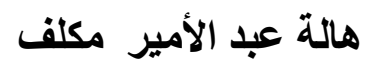

$$
\begin{aligned}
& \text { المديرية العامة لتربية بغداد/الكرخ الثانية ـ قسم الارشير ملفي التربوي } \\
& \text { amdrh796@gmail.com }
\end{aligned}
$$

DOI: https://doi.org/10.36231/ coedw/vol30no3.9

Received 7/4/2019, Accepted 30/6/2019

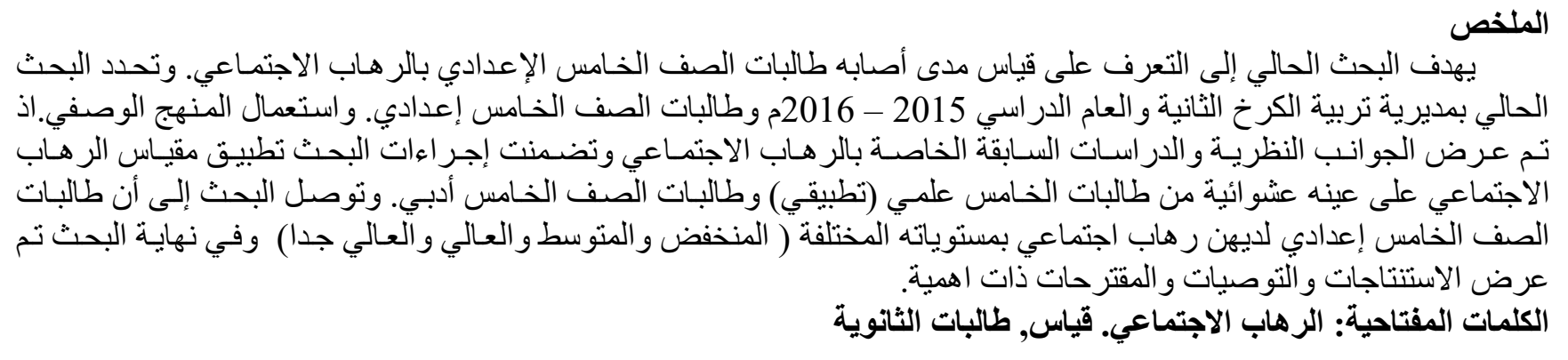

\title{
Measuring the Social Phobia Fifth-Secondary Stage Female Students
}

\author{
Hala Abdulameer Muklif
}

General Directorate of Education in Baghdad / Al-Karkh II / Department Of Education

\author{
Guidance
}

\begin{abstract}
The current research aims to know the measurement of the fifth- secondary stage female students' affliction with the social phobia. The research is confined to General directorate of Education in Baghdad / Al-Karkh/2 / for the academic year 2015-2016 and fifth-secondary stage female students. It has been used descriptive method and exposed theoretical sides and previous studies concerning with the social phobia.The procedures of the research include the application of the social phobia scale on random sample of fifth- secondary stage female students ( both branches scientific and literary). The current study has concluded that the fifth- secondary stage female students have phobia with different levels ( high, very high, medium and low) The current study has displayed singnificant conclusions, recommendations and suggestions.
\end{abstract}

Keywords: Social Phobia, Measuring, secondary female students 


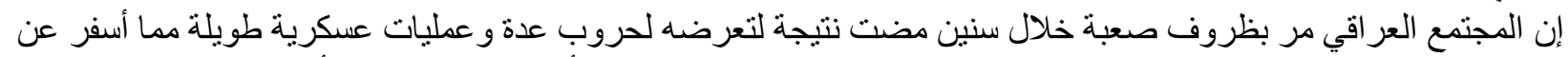

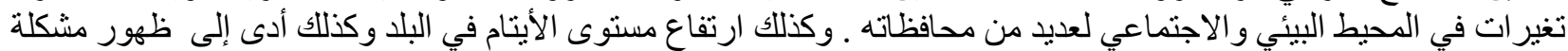

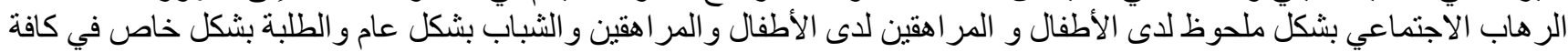

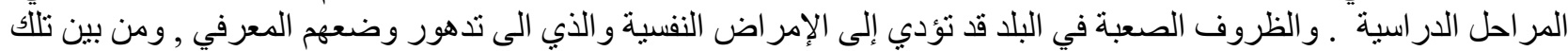

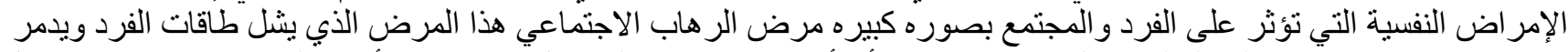

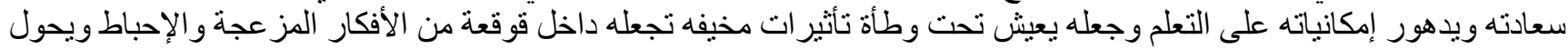

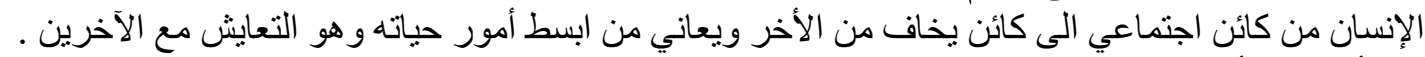

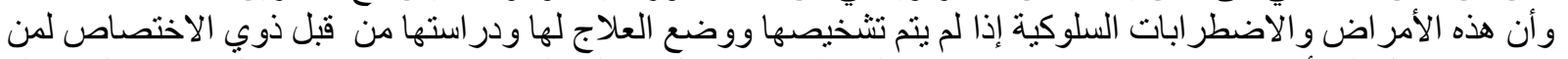

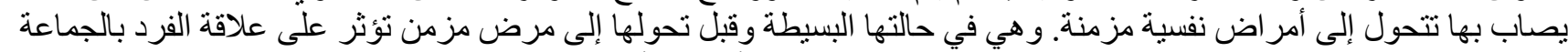

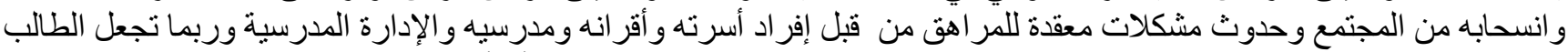

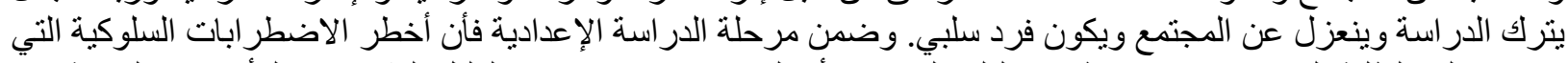

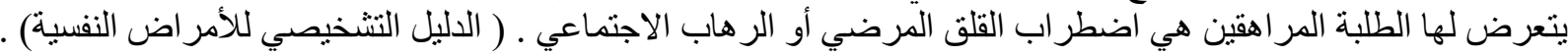

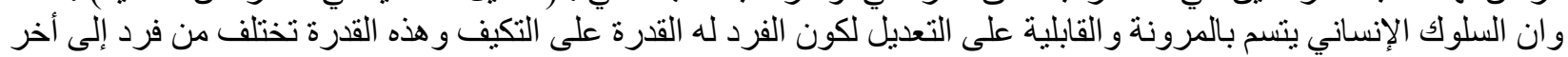

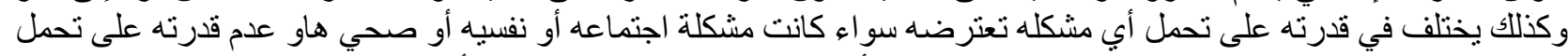

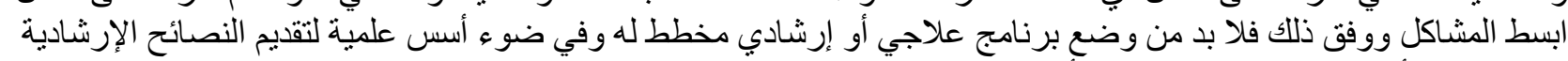

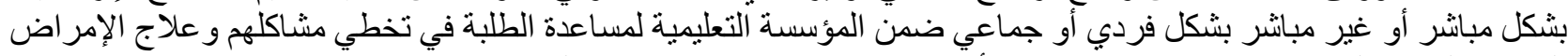

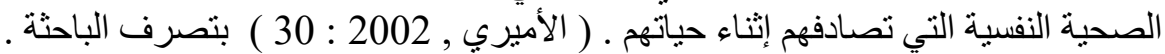

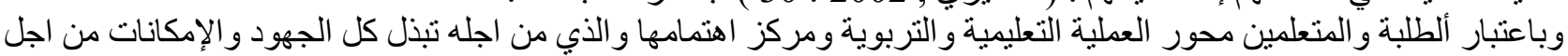

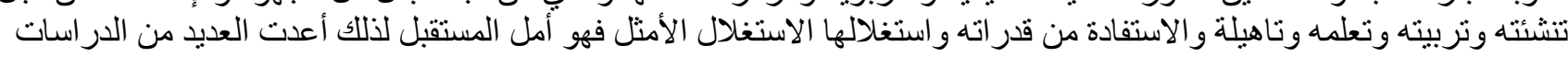

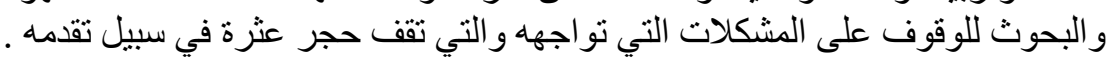

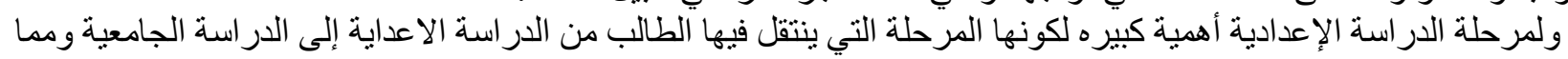

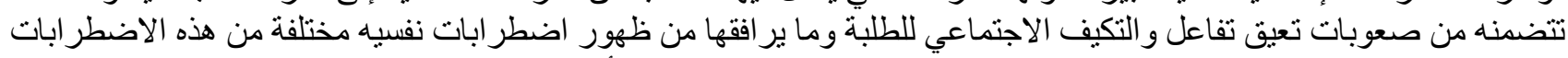

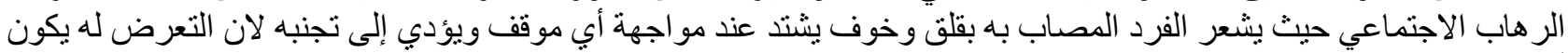

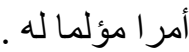

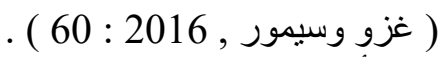

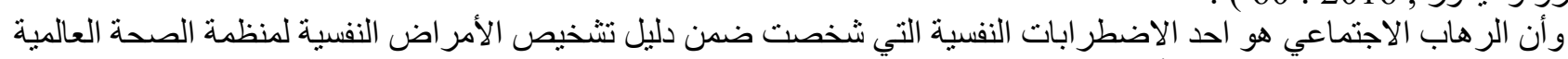

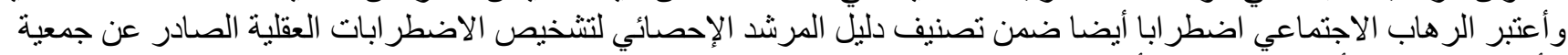

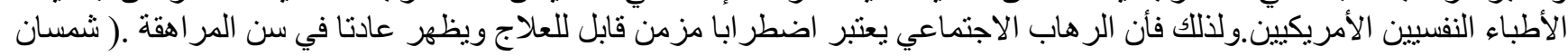

(2004،

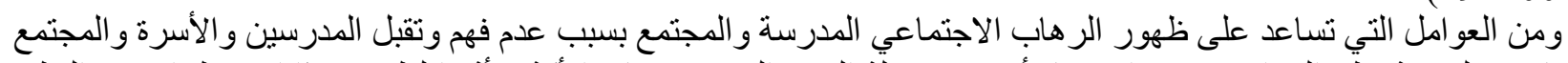

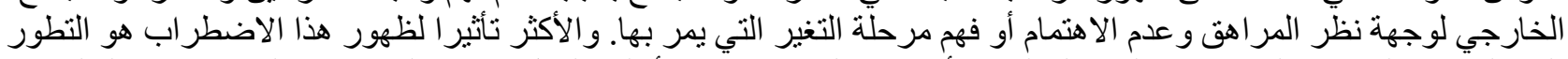

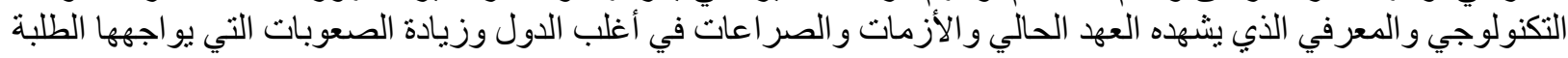
وخاصتا طُالبات المرحلة الإعدادية.

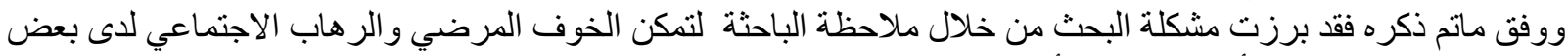

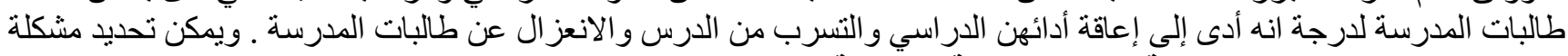

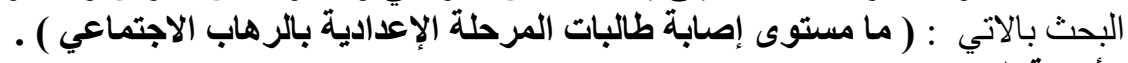
أهمية البحث الاني

تنبع أهمية البحث البحالي من أهمية الطلبة وأهمية دور هم في المجتمع فالطلبة ذو الدور المنشود في بناء البلد ونهوضـه وهم جيل

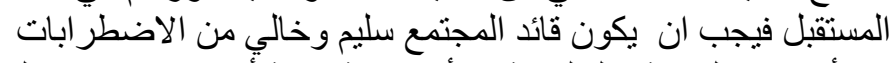

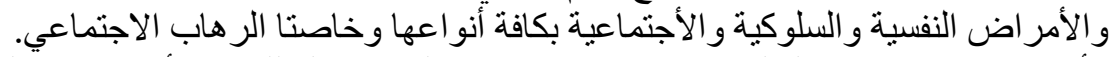

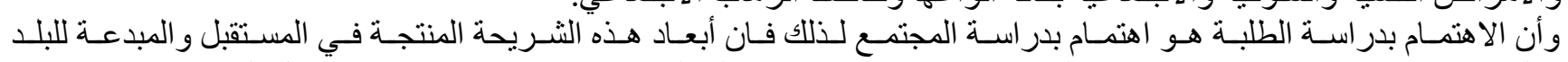

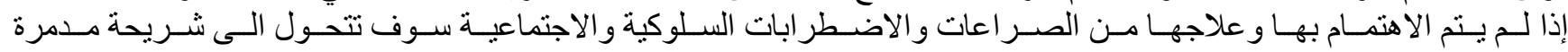

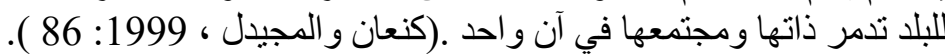

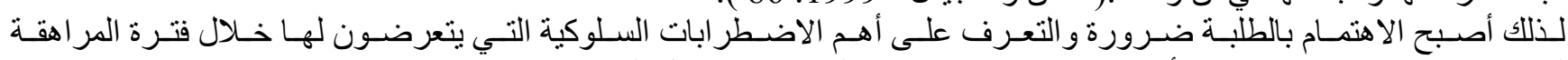

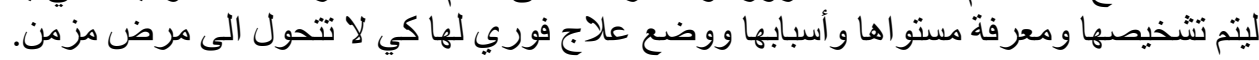




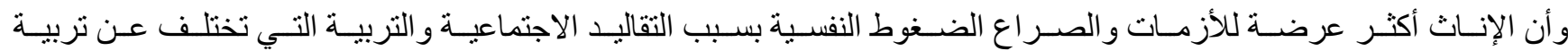

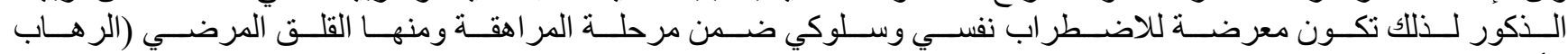

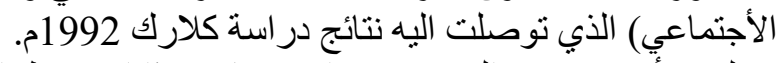

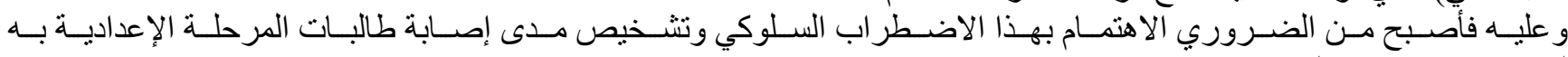
لوضع علاج مناسب لـ له. له.

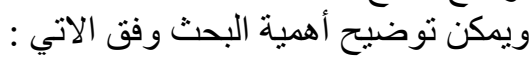

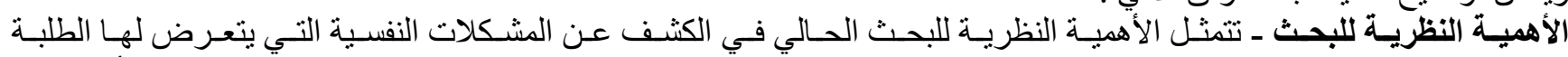

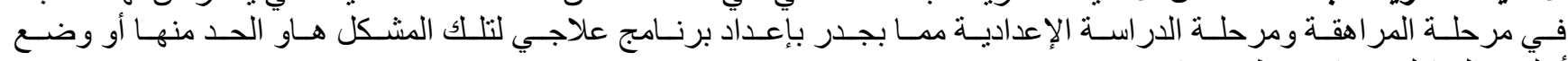

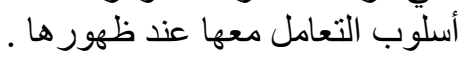

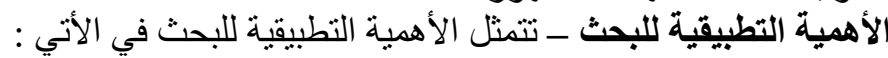

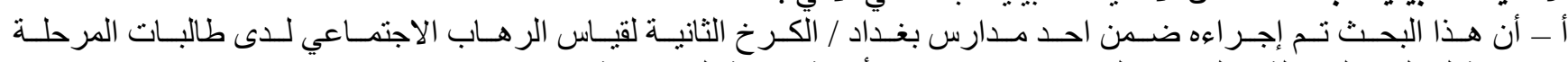

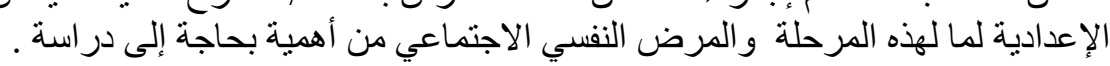

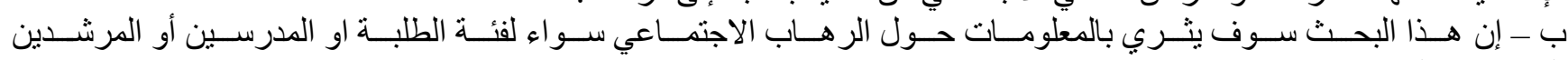

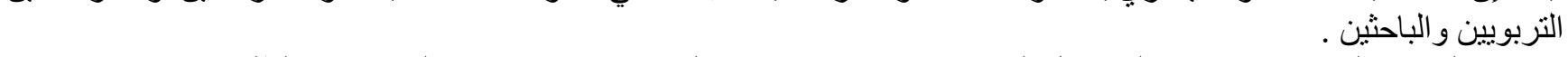

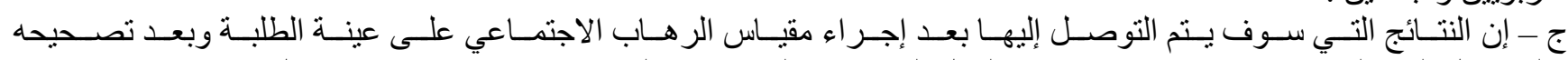

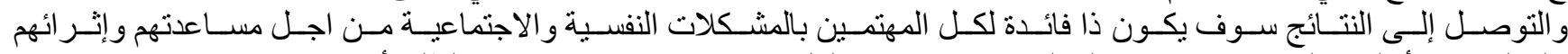

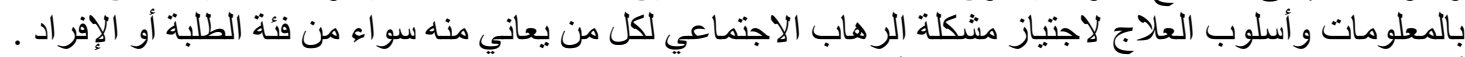

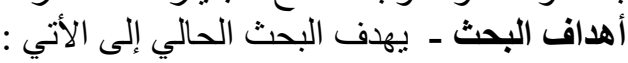

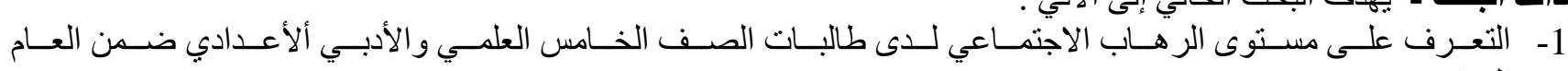

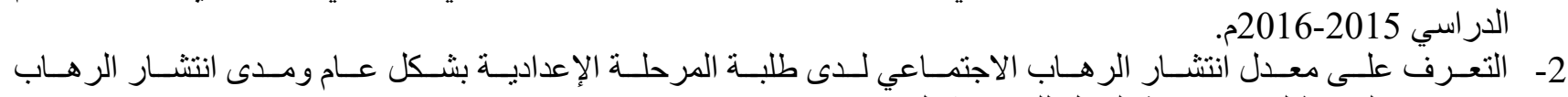

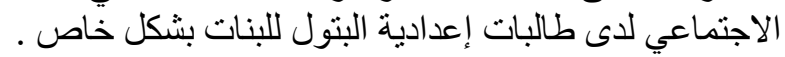

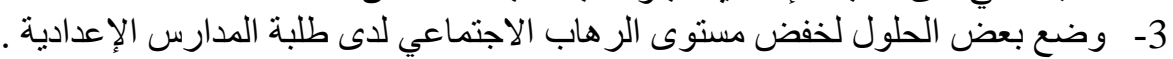

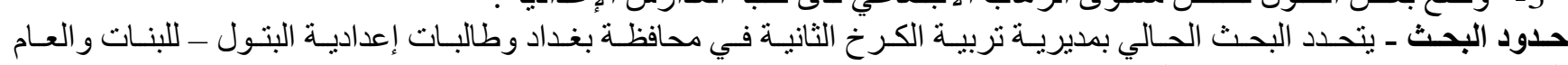

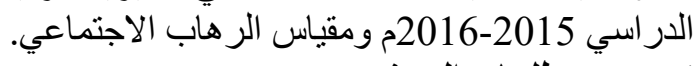
تحديد مصطلحات البحث

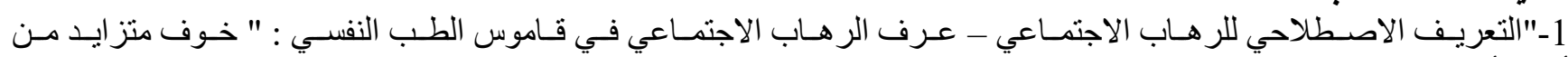

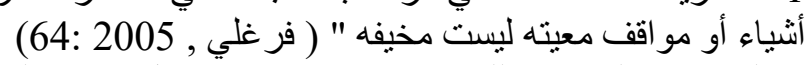

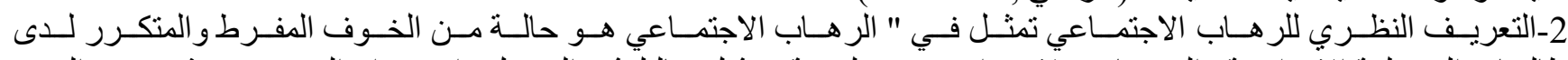

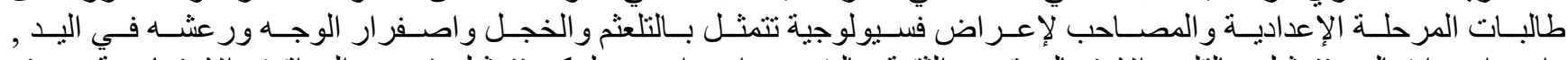

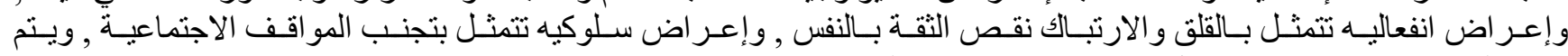

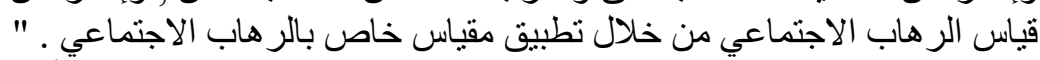

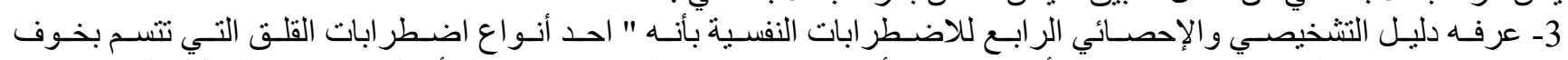

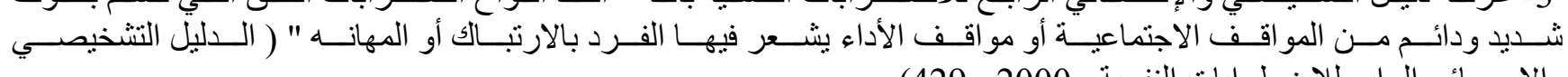

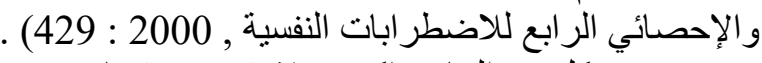

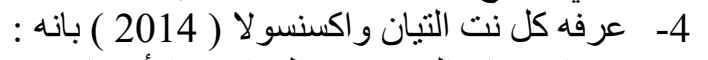

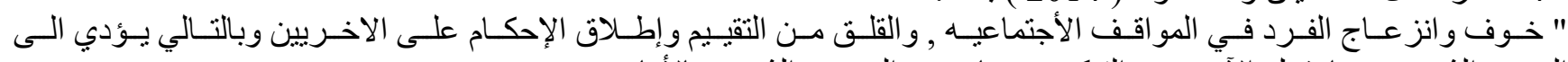

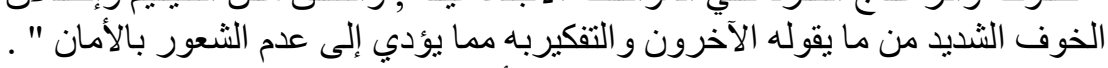

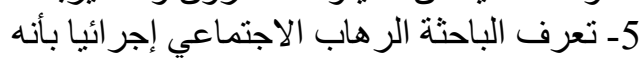

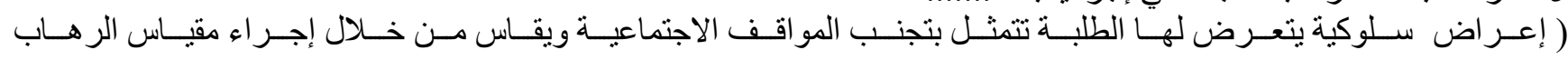

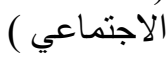

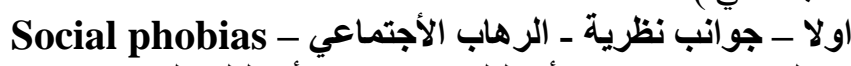

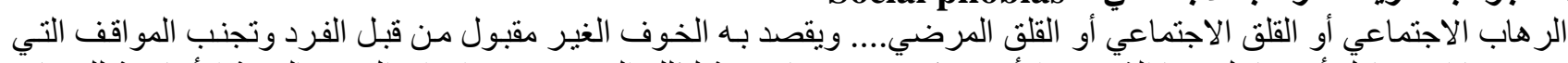

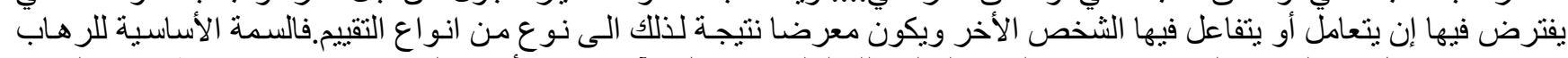

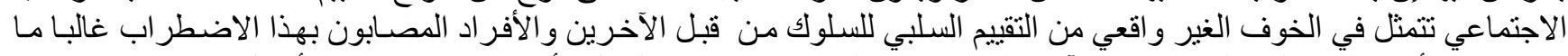

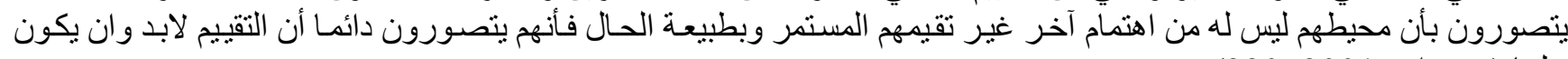

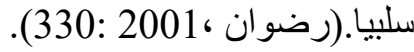
ويظهر الرهاب الاجتماعي لدى الأفراد عادة في سن المر اهقة ويتمركز حول الخوف من أن يكون الثخص محط أنظـار الآخرين في

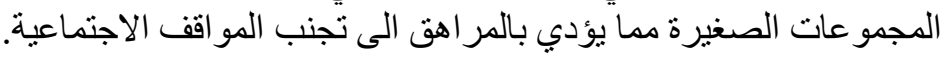


ويصاب الرجال و النساء بالر هاب الاجتماعي لكن النساء يكون مستوى الإصابة أكثر من الرجال حسب مـا أكدت علبه دراسـة كلارك 1992. ودر اسة الريامي 2010.

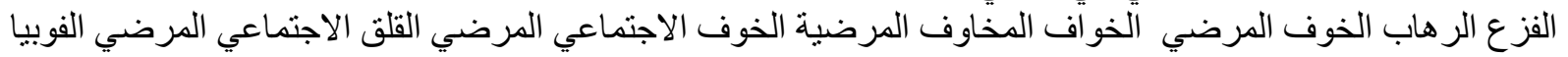

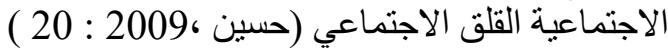

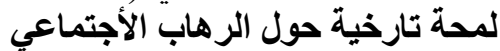

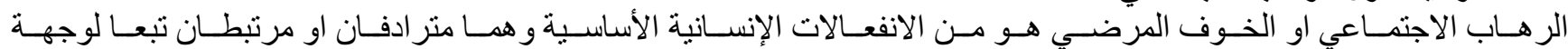

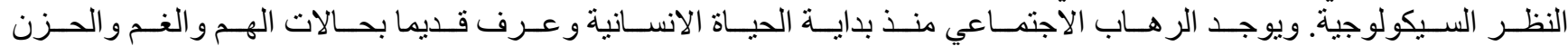

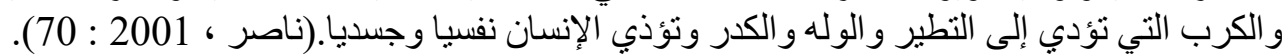

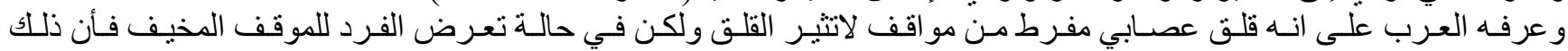

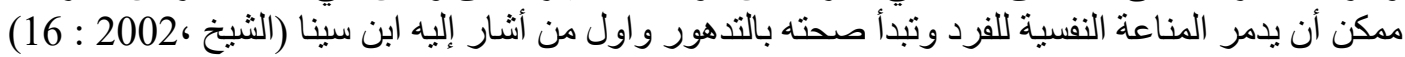

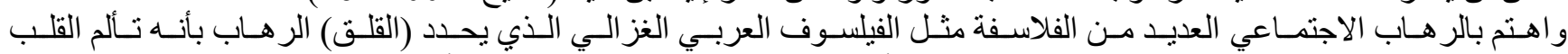

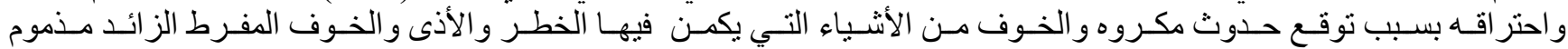

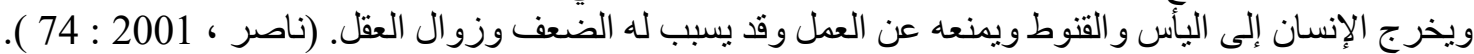

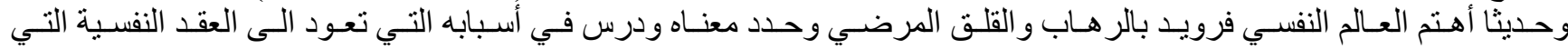

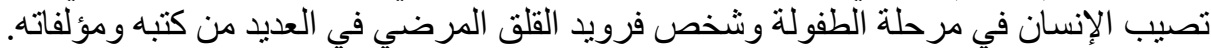
الأسس النظرية للزرهاب الأجتماعي

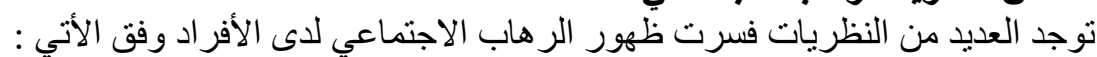

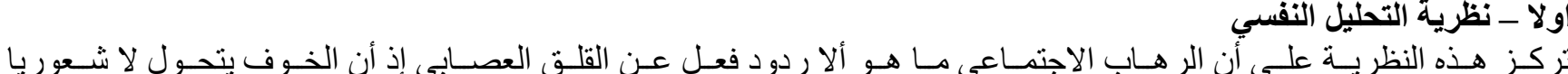

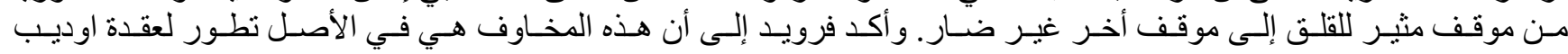

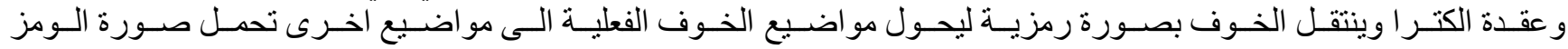

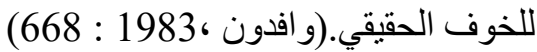

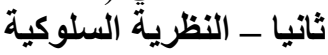

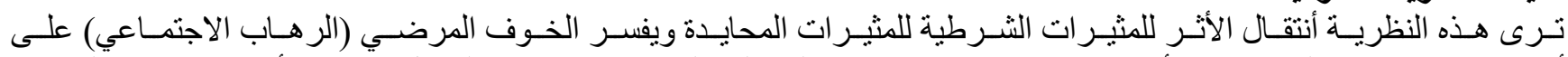

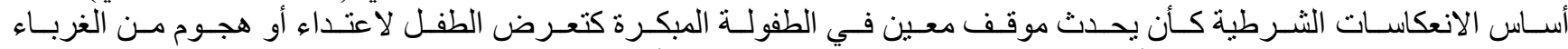

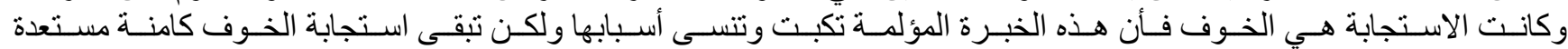

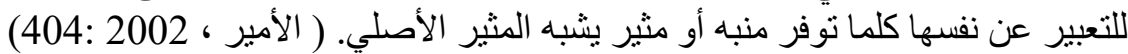

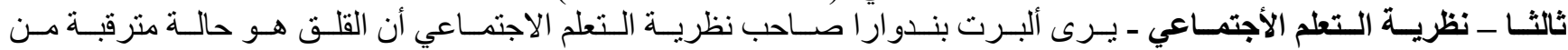

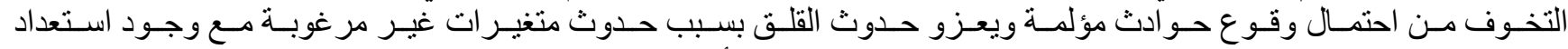

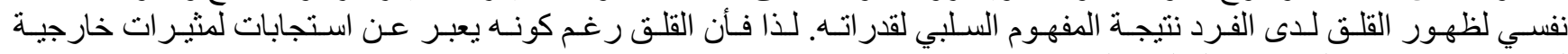

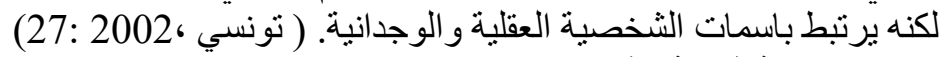

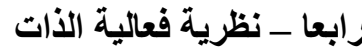

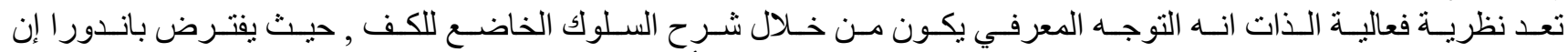

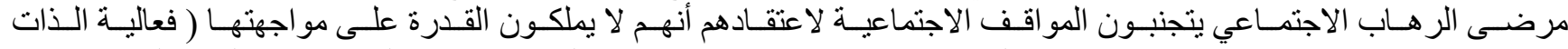

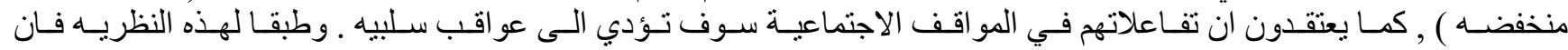

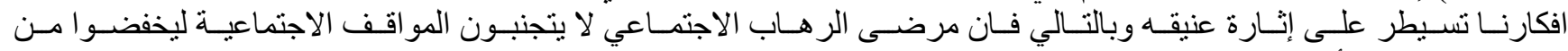

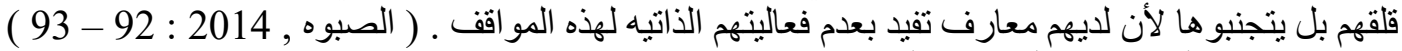

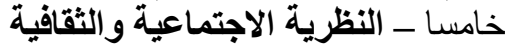

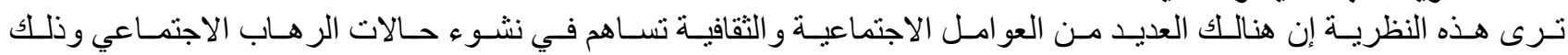

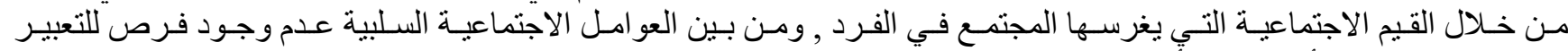

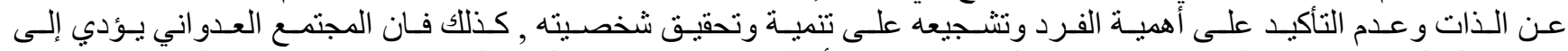

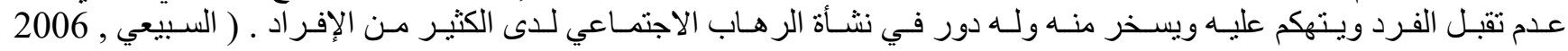

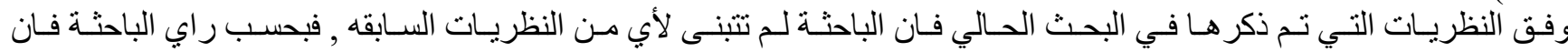

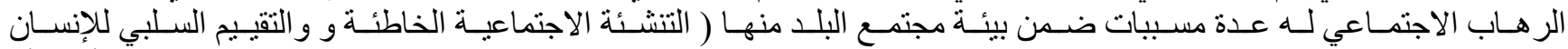

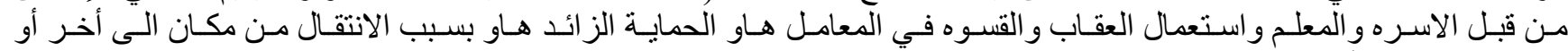

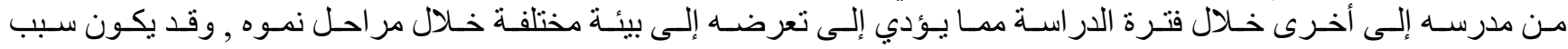

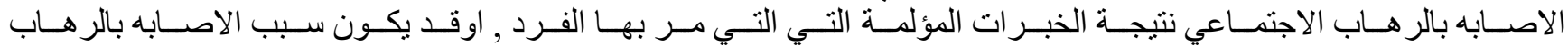




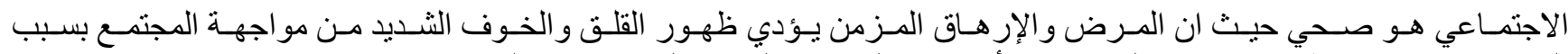

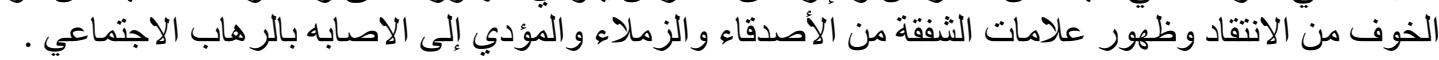

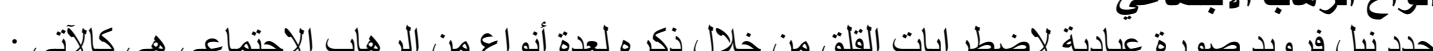
انواع الرهاب فرويد الاجتماعي

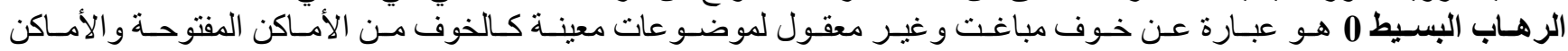

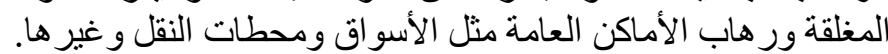

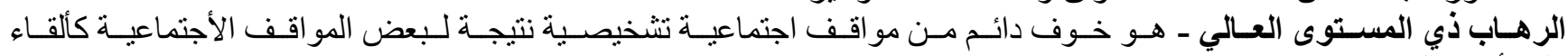

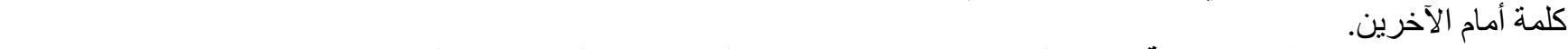

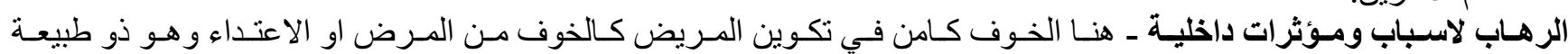

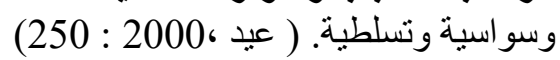

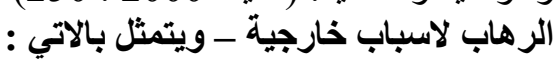

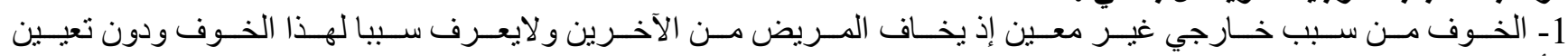

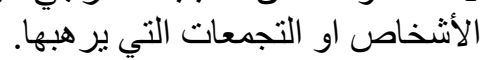

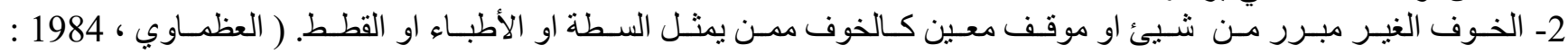

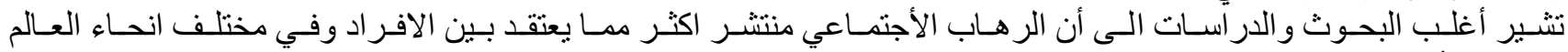

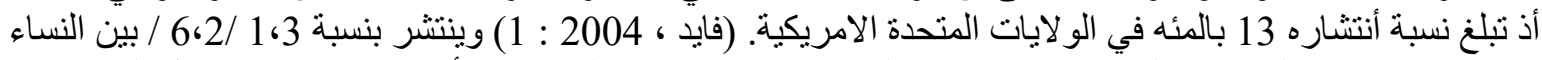

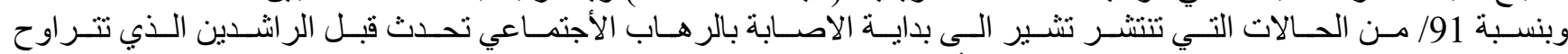

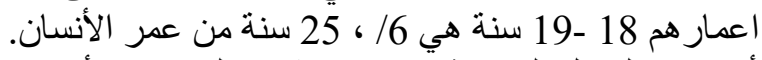

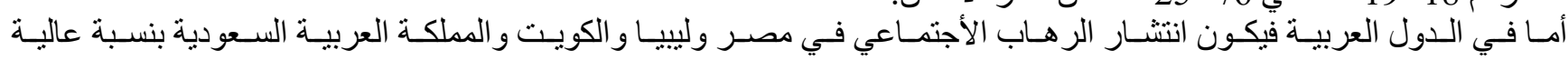

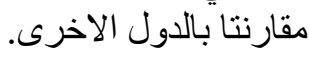

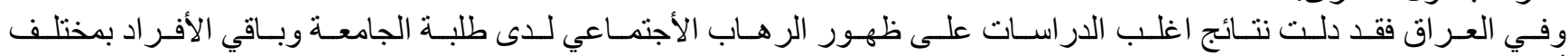

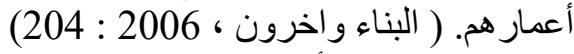

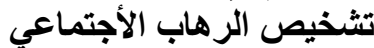

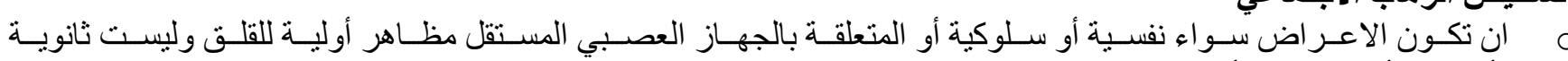
لأعر اض أخرى مثل الأفكار الوسواسية.

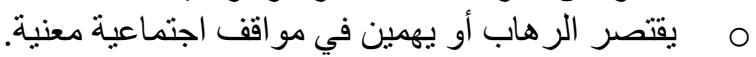

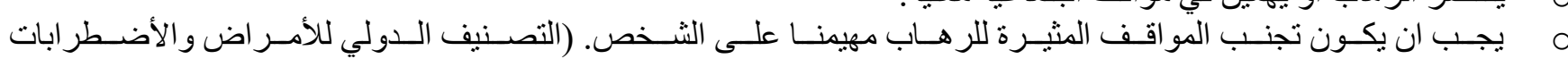
النفسية ، 1999 : 147 : و استتادا على تحديد طبيعة الرهاب يمكن تشخيص الفرد المصاب بالرهاب الأجتماعي يكون مصاب بالأعر اض الاتيه : أعراض نفسية

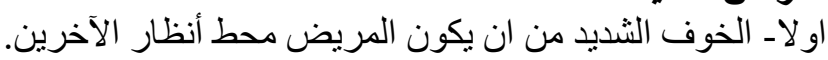

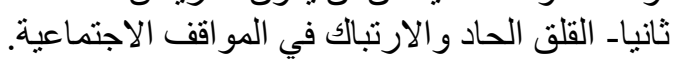

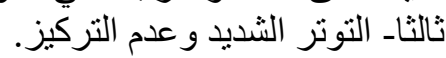

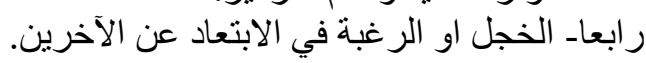

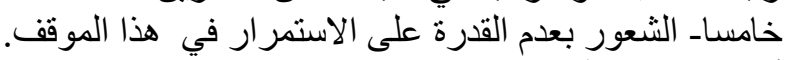

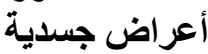

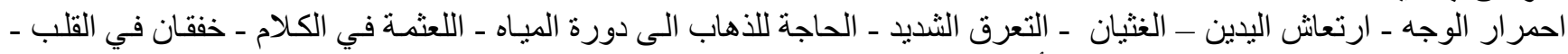

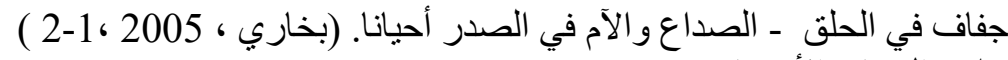
علاج الرهاب الأجتماعي

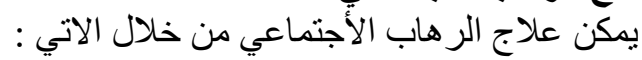

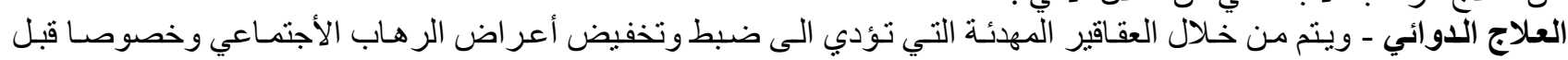

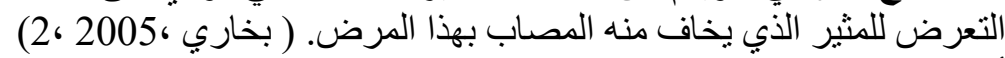

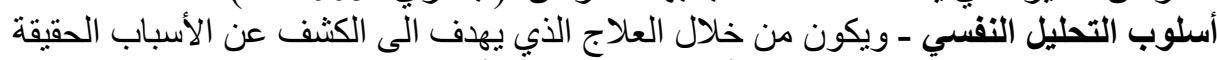

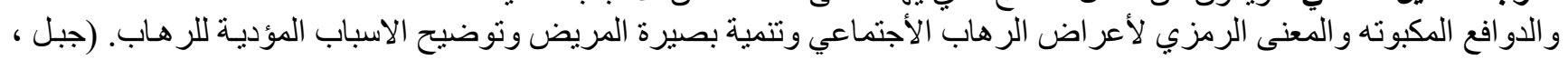




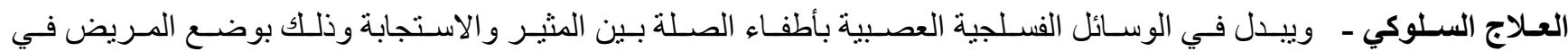

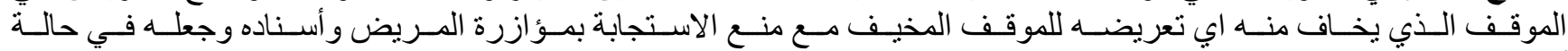

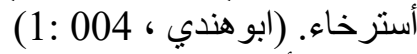

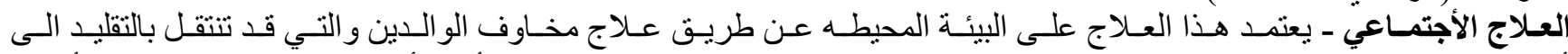

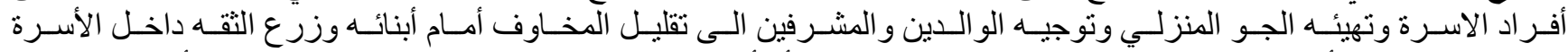

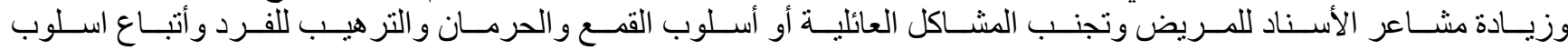
التشجيع و المساندة و الثواب. (جبل ، ثانيا- دراسات سابقة ـ أ- دراسات عربية

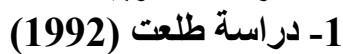

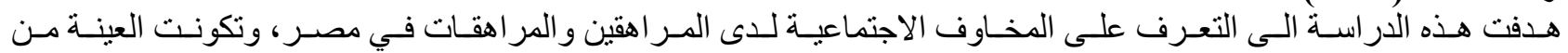

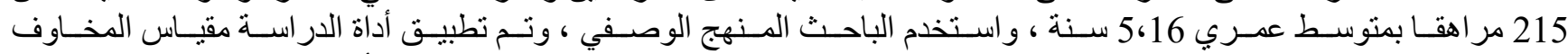

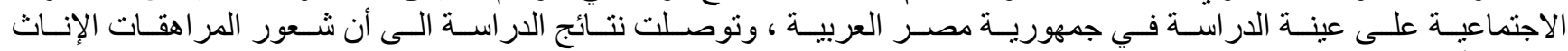
بالخوف أكبر من شعور المر اهقين الذكور . (طلعت ،1992)

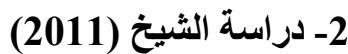

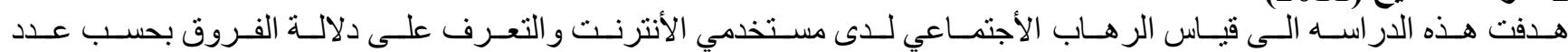

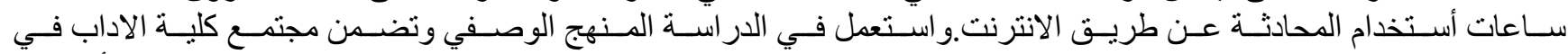

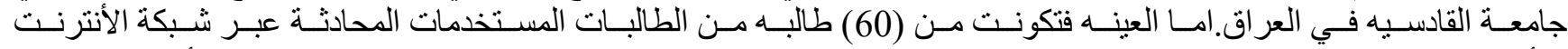

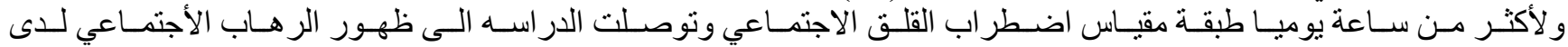

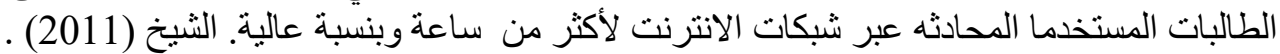

ثُاتيا الاراسات الأجنبيه 1- دراسة باستيا ولويوريو الأبيه 2012

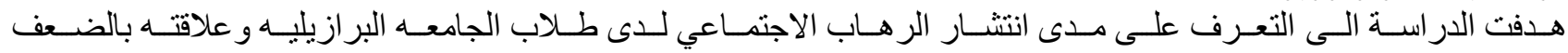

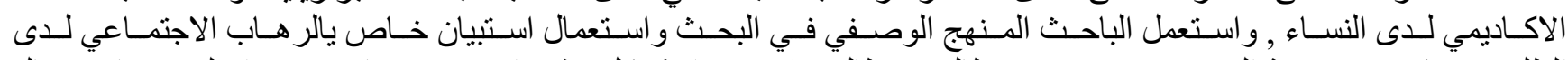

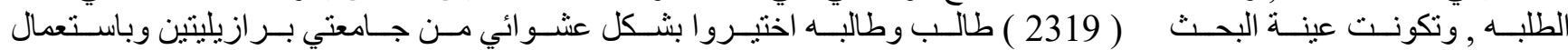

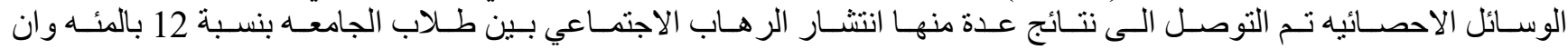

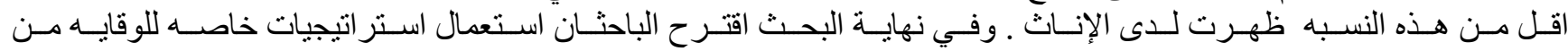

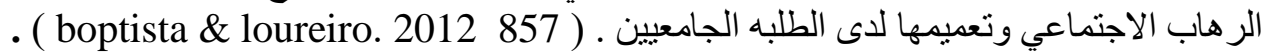

2- دراسة كوركين ووينستر 2014

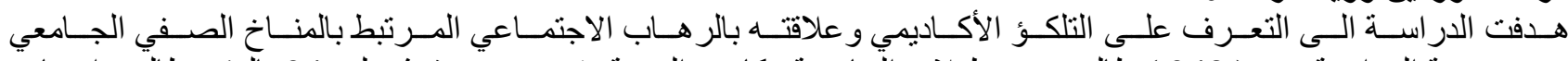

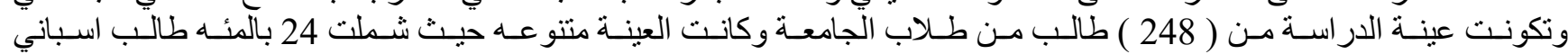

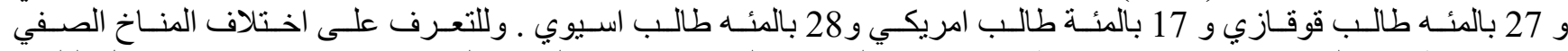

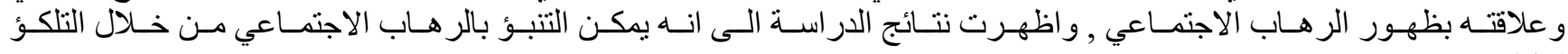

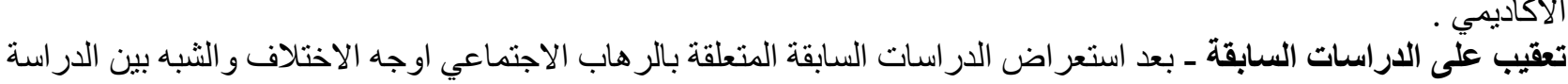

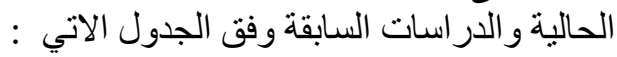

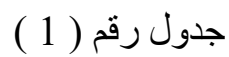

لتوضيح اوجه الاختلاف و الثبه بين الدراسة الحالية وبين الدر اسات السابقه

\begin{tabular}{|c|c|c|c|}
\hline اوجه الاختلاف & أوجه الشبه & الاختلاف & الاجراء \\
\hline 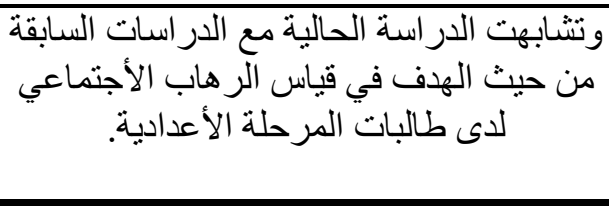 & 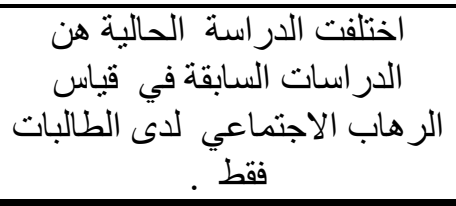 & 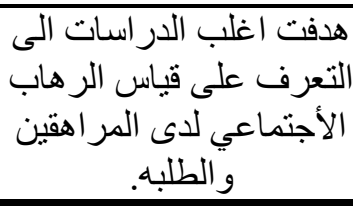 & اللهذف \\
\hline 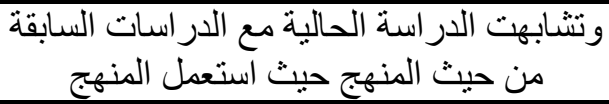 & لاليوجد اختلاف من حيث النستة التعمال & اتبع في جميع الدراسات الوصفي & المنهج \\
\hline
\end{tabular}




\begin{tabular}{|c|c|c|c|}
\hline الوصفي. & مقارنة بالدر اسات السابقة & & \\
\hline 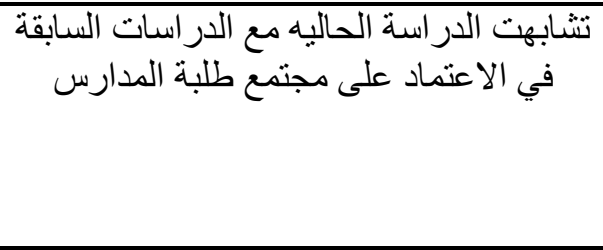 & 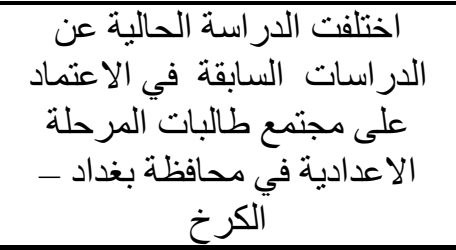 & 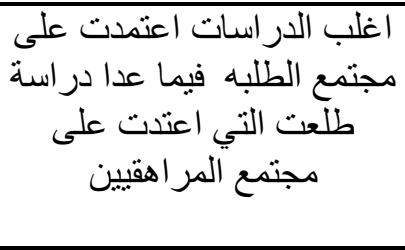 & المجتمع \\
\hline 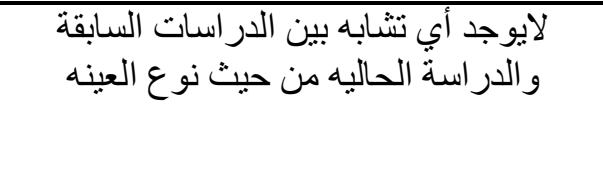 & 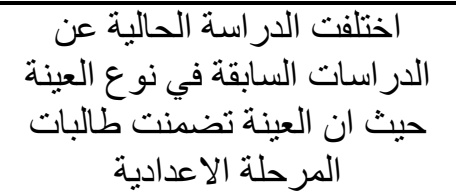 & الدر اسات على عبنة في جميعة الجامعة & العينه \\
\hline ولايوجدة أي تثشابه بين الدر اسات الدات السابقة & 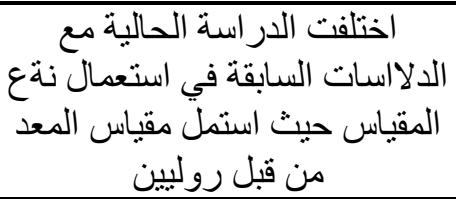 & 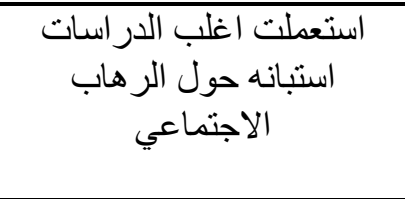 & أداة البحث \\
\hline 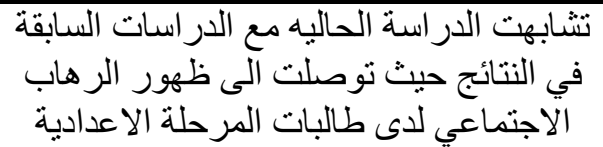 & 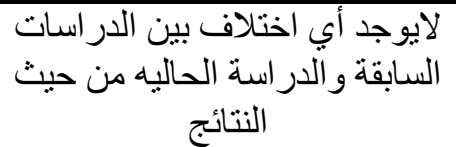 & توصلت الى ظهور الرهات السابقة & النتائج \\
\hline
\end{tabular}

$$
\begin{aligned}
& \text { الفصل الثالث ـ اجراءات البحث } \\
& \text { اولا- منهج البحث المث الته }
\end{aligned}
$$

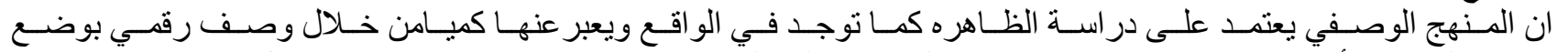

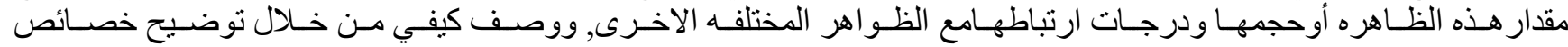

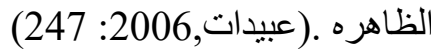

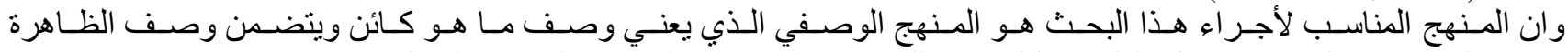

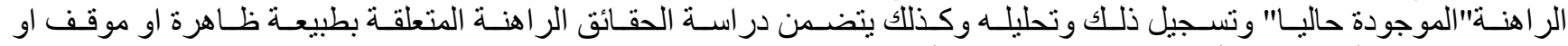

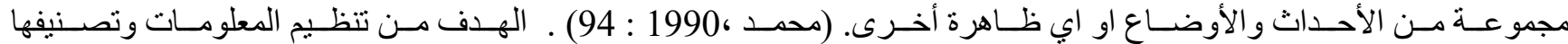

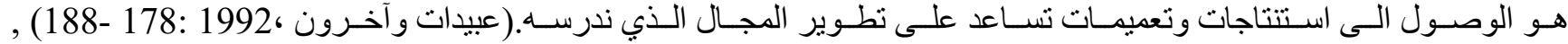

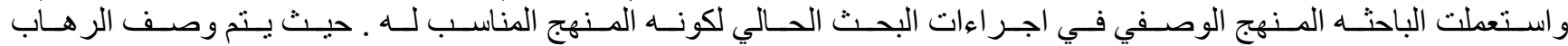

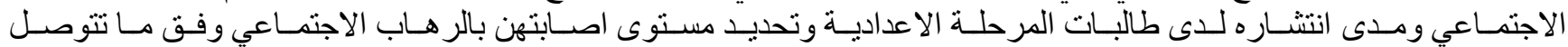

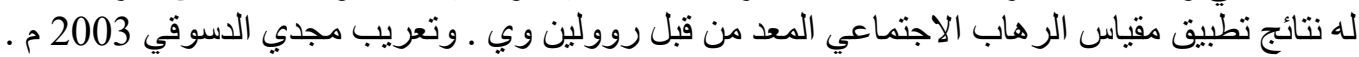

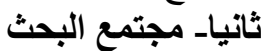
يمثل مجتمع البحث البحث الحالي طالبات اعدادية البتول للبنات الو اقعة في منطقة الدورة ضمن قاطع الكرخ الثانية من محافظة بغداد. وشمل

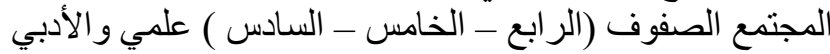
المستمر ات بالدوام ضمن العام الدر اسي 2015 -2016م. البالغ عددهن (778) طالبه. وكما يوضحه الجدول الاتي بشكل مفصل:

\begin{tabular}{|c|c|c|c|c|c|c|c|}
\hline عدد الطالبات & الشعبة & الفرع & الصف & عدد الطالبات & الشعبه & الفرع & الصف \\
\hline 40 & أ & \multirow{6}{*}{ أدبي } & \multirow[t]{3}{*}{ الرابع } & 48 & أ & \multirow{6}{*}{ علمي } & \multirow[t]{3}{*}{ الرابع } \\
\hline 42 & ب & & & 49 & ب & & \\
\hline 43 & ج & & & 49 & ج & & \\
\hline 40 & أ & & \multirow[t]{3}{*}{ الخامس } & 33 & أ & & \multirow[t]{3}{*}{ الخامس } \\
\hline 30 & ب & & & 39 & ب & & \\
\hline 37 & ج & & & 46 & ج & & \\
\hline
\end{tabular}

$$
\text { جدول رقم (2) يوضح مجتمع البحث }
$$



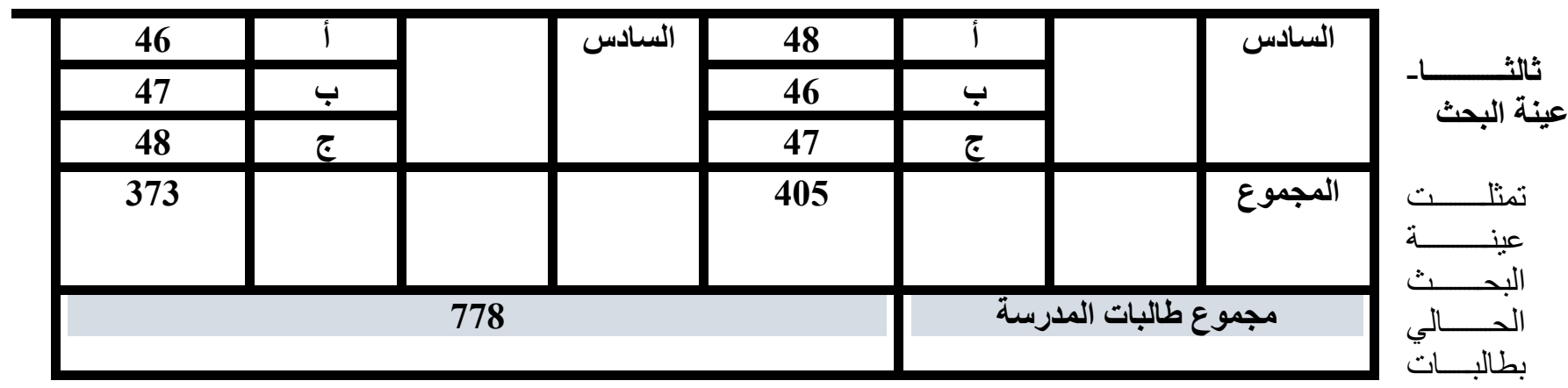

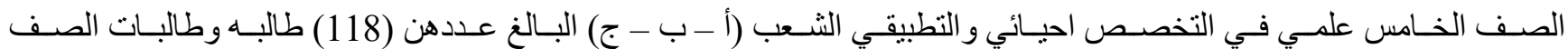

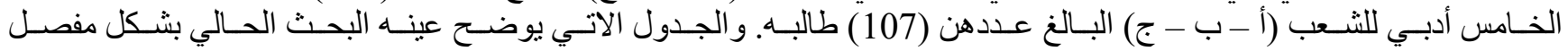

جدول رقم (3) يمثل عينة البحث

\begin{tabular}{|c|c|c|c|c|c|}
\hline المجموع & عدد الطالبات & الشعبة & التخصص & الفرع & الصف \\
\hline \multirow{3}{*}{118} & 33 & أ & أحيائي & \multirow{3}{*}{ علمي } & \multirow{6}{*}{ إعدادي } \\
\hline & 39 & ب & & & \\
\hline & 46 & ج & تطبيقي & & \\
\hline \multirow{3}{*}{107} & 40 & i & \multirow{3}{*}{ أدبي } & \multirow{3}{*}{ أدبي } & \\
\hline & 30 & ب & & & \\
\hline & 37 & ج & & & \\
\hline 225 & 225 & 6 & 3 & 2 & المجموع \\
\hline
\end{tabular}

رابعا - أداة البحث

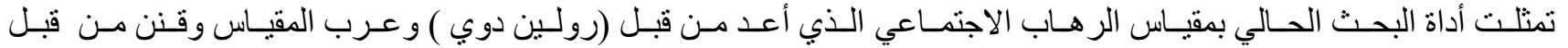

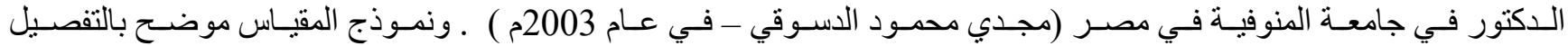

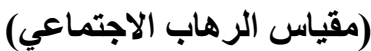
اعداد : رولين ووي الرهاب الاجنماعي) \& wee (1994lin \& wee) 0 تعريب وتقنين دكتور / مجدي محمد الدسوقي 2003م

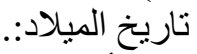

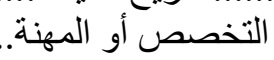

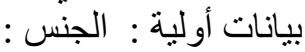

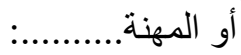

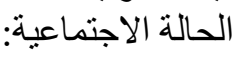

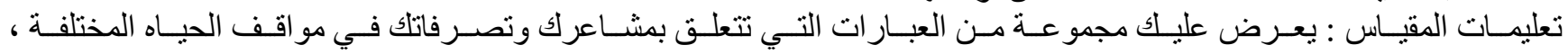
ويوجد امام كل عبارة اختيار ان هما نعم ، و والا.

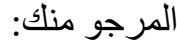

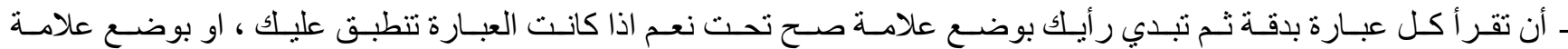
صح تحت ال اذا كانت العبارة ال تنطبق عليك.

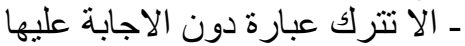

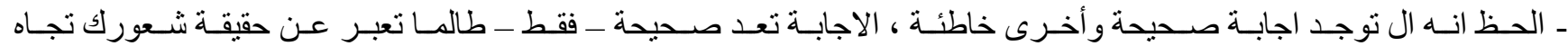

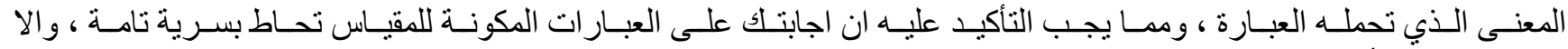

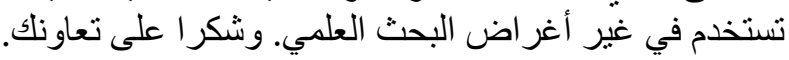

1. احب البقاء في الفراش حتى ال أرى أي شخص.

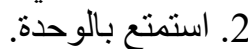

3.أفضل عادة ان اكون في صحبة الاصدة ألفاء على أن أكون وحيدا.

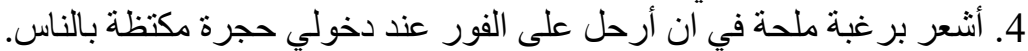

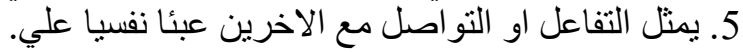

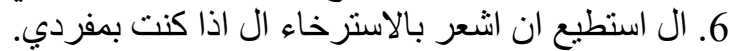

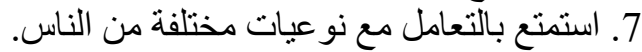
8. أبتعد عن الاخرين قدر الامكان. 
9. أمارس هوياتي المفضلة بمفردي.

10. أخبر الاخرين انني لست على الإنى ماير ام التجنب مشاركتهم القيام ببعض المهام. 11. أشتعر بالارتياح عندما اكون بمفردي.

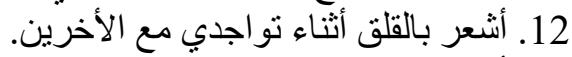

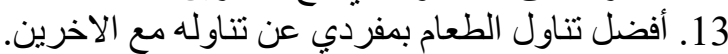

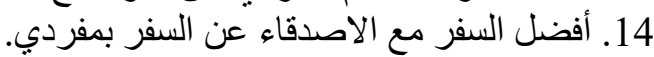
15. أفضل الذهاب الى السينما بمفردي. 16. 16 أستمتع دائما بوجودي الفي مع الأخرين. 17. أفضل الخروج مع الأصدقاء عن الجلوس الأنس وحدي بالبيت.

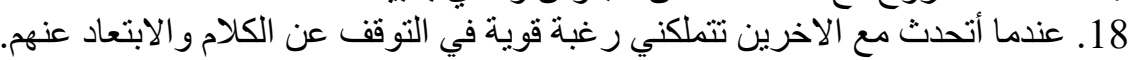

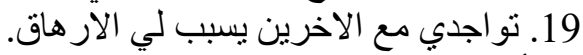

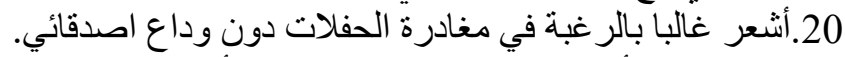

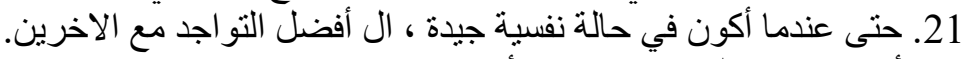

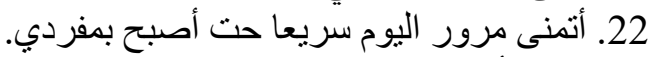

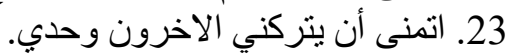
24. أشعر بالأمان عندما أكون بمفردي التروني.

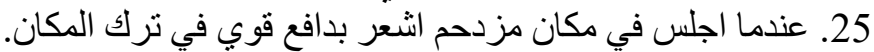
26. احتاج أن أكون بمفردي تماما لعدة أيام.

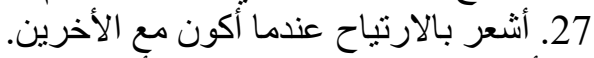

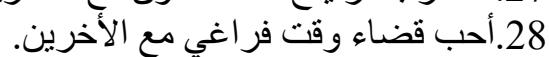
29. عندما أقرر أن أكون مع الأخرين اندم على ذلى اللك فيما بعد.

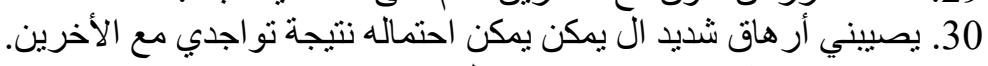

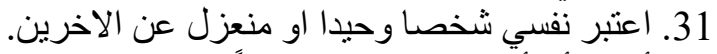

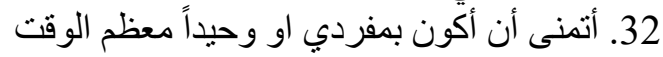

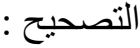

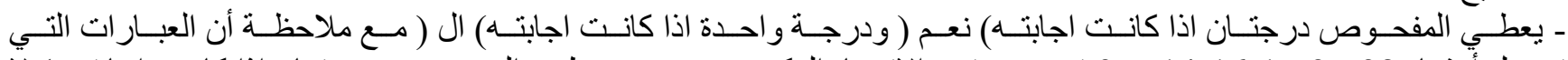

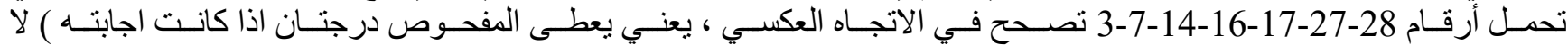
ودرجة واحدة اذا كانت اجابته نعم.

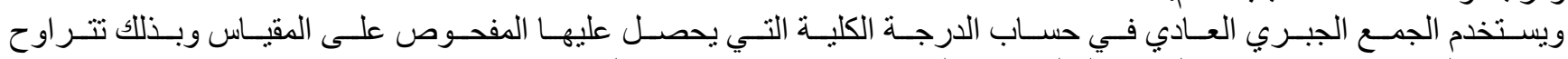
در اجات المقياس بين 64-1 - ـ الدرجة التئ العالية تشير الى خوف اجتماعي مرتفع و العكس صحيح. صدق أداة البحث

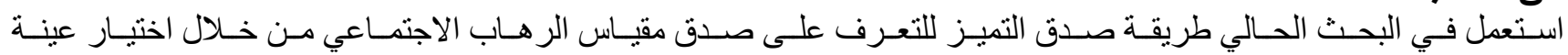

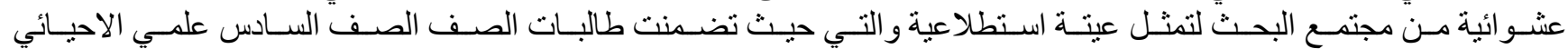

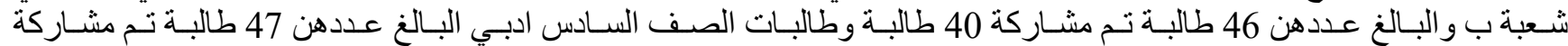

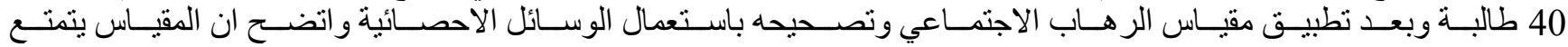
بصدق تميزي عالي وكما موضح في الجدول الاتي :

\begin{tabular}{|c|c|c|c|c|c|c|c|c|c|c|}
\hline \multirow{2}{*}{ النتيجير } & \multirow{2}{*}{ مستوى } & \multirow{2}{*}{ الحريدة } & \multicolumn{2}{|c|}{ قيمة ت } & \multirow{2}{*}{ الالمعرافياري } & \multirow{2}{*}{ الحسبابي } & \multicolumn{2}{|c|}{ عدد الطالبات } & \multirow[t]{2}{*}{ الشعبة } & \multirow{2}{*}{ والفرع } \\
\hline & & & الحدولية & المحسوبة & & & المشاركات & الكلي & & \\
\hline \multirow{2}{*}{ بتمبيز } & \multirow[t]{2}{*}{0,05} & \multirow[t]{2}{*}{79} & \multirow[t]{2}{*}{1.99} & \multirow[t]{2}{*}{1.587} & 7.89 & 40 & 40 & 46 & ب & \\
\hline & & & & & .7 & 39 & 40 & 47 & ب & أدبي \\
\hline
\end{tabular}




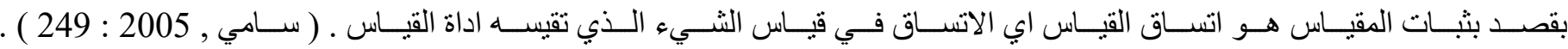

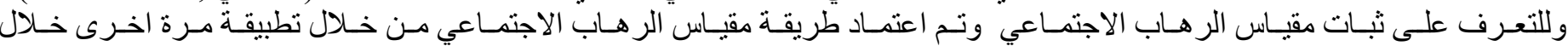

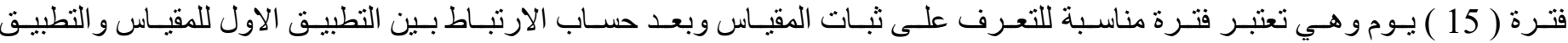

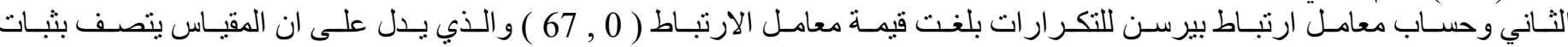

خامسا - تطبيق أداة البحث

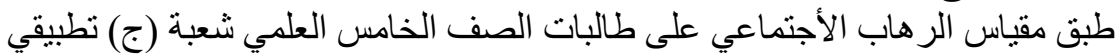

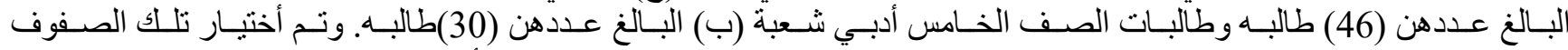

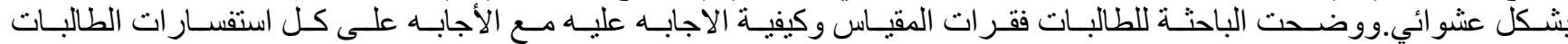

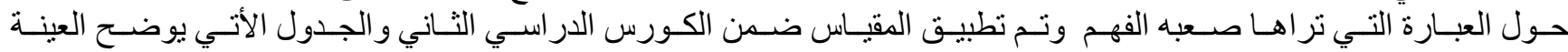

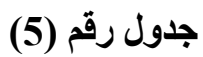
التي طبق عليها المقياس بشكل مفصل: يوضح العينه التي طبق عليها مقياس الرهاب الاجتماعي

\begin{tabular}{|c|c|c|c|c|}
\hline عدد الطالبات & الشعبة & التخصص & الفرع & الصف \\
\hline 46 & 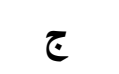 & تطبيقي & علمي & الخامس اعدادي \\
\hline 30 & ب & الدبي & البي & \\
\hline 76 & 2 & 2 & 2 & المجموع \\
\hline
\end{tabular}

اولا - عرض نتائج البحث

بعد تطبيق مقياس الرهاب الأجتماعي وتصحيحها بالجمع الجبري تم التبات التوصل نتائج مختلفة تم ادر اجها بشكل مفصل

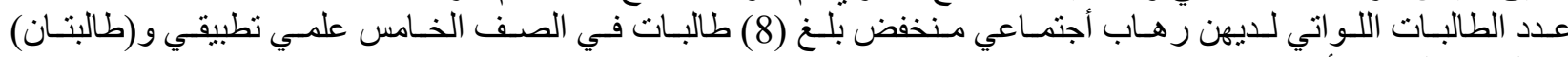

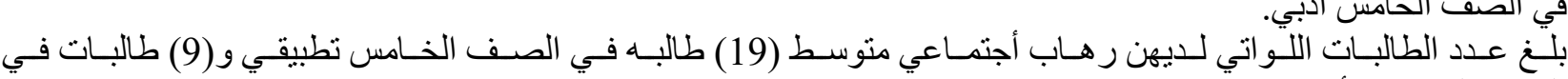
الصف الخفامس الأدبي.

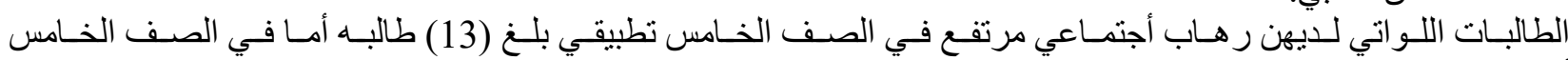
أدبي فقد بلغ (12) طالبه.

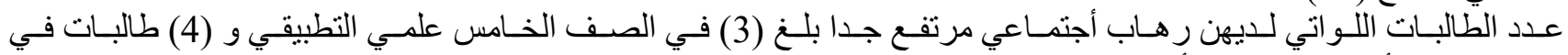

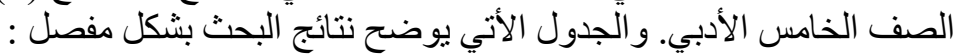
جدول رقم (6) يوضح نتائج البحث البش

\begin{tabular}{|c|c|c|c|c|c|c|c|c|c|}
\hline الصجموع & الطالبات & الطالبات & جتماعي & هاب الماب & طالبات بـ & عدد اصاب & الفرع & الصف & 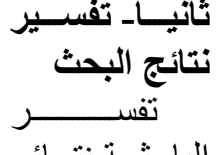 \\
\hline & & & مرتفع & مرتفع & متوسط & منخفض & & & ي توصـلت \\
\hline 46 & 3 & 43 & 3 & 13 & 19 & 8 & (تطبيقي) & أعدادي & 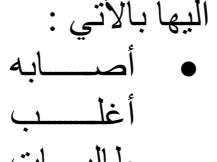 \\
\hline 30 & 3 & 27 & 4 & 12 & 9 & 2 & ادبي & & 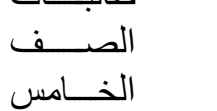 \\
\hline
\end{tabular}




$$
\begin{aligned}
& \text { علمي و الادبي بالر هاب الاجتماعي. }
\end{aligned}
$$

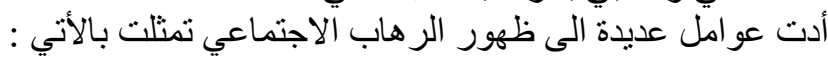

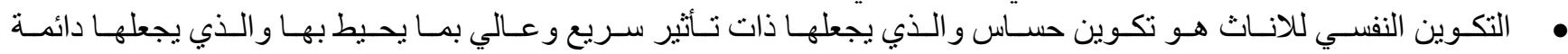

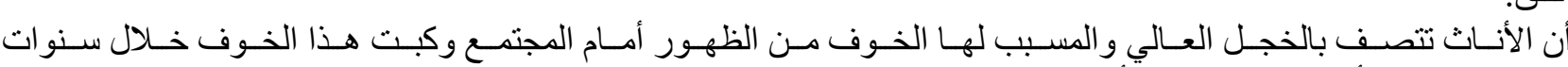

$$
\begin{aligned}
& \text { حياتها يؤدي الى أصابتها بالر هاب الأجنماعي. }
\end{aligned}
$$

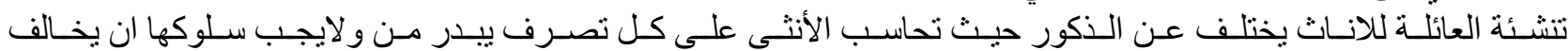

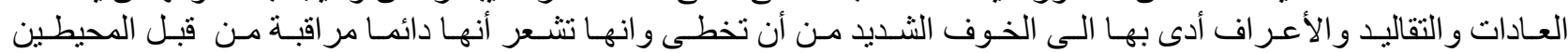

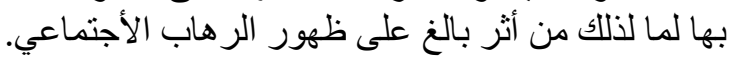

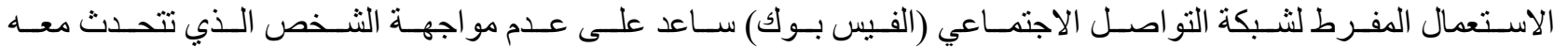

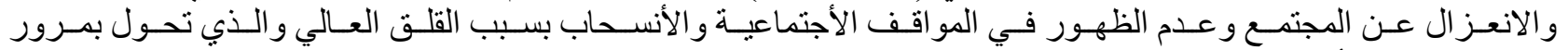

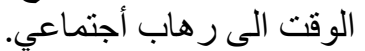

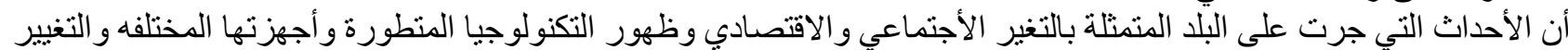

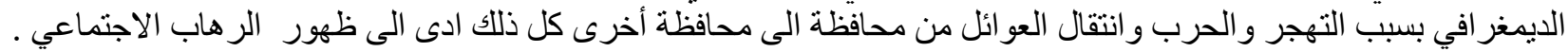

\section{Recommendations and Conclusions \\ Conclusions}

In this current research, a set of conclusions have been made:

1- Social anxiety disorder leads to emotional and psychological imbalance;fear,isolation, fear of others, dreadof participating in social events and communication with others.

2- Self-esteem means the value that the individual has for himself, and it is affected by thesurrounded environment and his safety from thepsychological and physicaldiseases, the proper individual has good self-esteem and feels confident of himself but the individual who has a behavioral disorderand social phobia usually has low self-esteem.

3- The parent's mistreatment of their children and exposing them to strict upbringing style and cruel treatment encourages the appearance of social phobia in the future to them.

4- The use of traditional learning by teachers in dealing with studentslike unaccepting theiropinions and not letting them express their thoughts, or using reprimanding style, student's admonition and punishment in front of their colleagues, all of these causes, effects on the student'spersonalityjudgment to do anything, inability to make decisions and lack of selfconfidence, which inturn helps to develop social phobia.

5- The use of behavioral therapy and cognitiveprograms by educational counselors helpsin the treatment of studentshow have social phobia.

\section{Recommendations}

According to the conclusions of this research, the researcher recommends the following:

1- Preparingspecial guide for educational counselors, teachers andlecturers thatillustrates the most important diseases of the modern era, and the diseases that are caused by psychological, social and economic crisis to students, children and teenagers, including behavioral disorder, high anxiety and social anxiety.

2- Arrangingtraining coursesat the Ministry of Education, including Baghdad educationaldirectorates, department of preparation and training, to train educational counselors on methods to detect social phobia, its causesand approaches of treatment.

3- Spreading health and psychological awareness among students through awareness fliers and postersabout the most important mental and psychological conditionsby putting them in schools, thatshows the psychological and social effects and the methods of treatment.

4- Holding seminars and lectures for teachers regarding the modern methods to deal withteenager's students, and the most important problems and behavioral disorders that they are exposed to, and to avoid the intimidation and panic styles that helps the emergence of social phobia. 


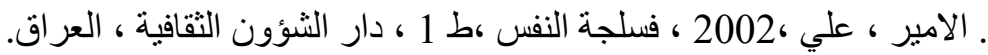

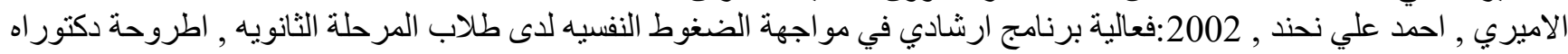

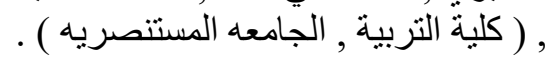

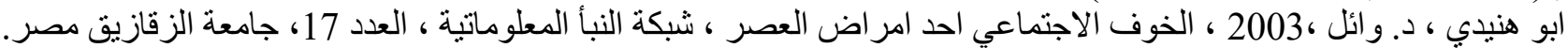

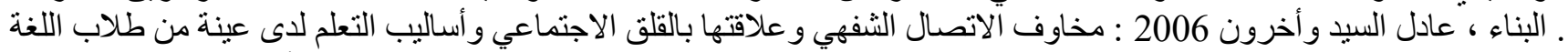

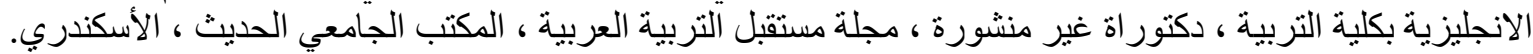

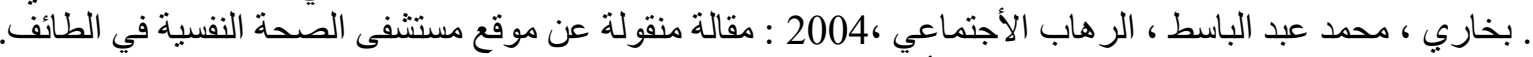

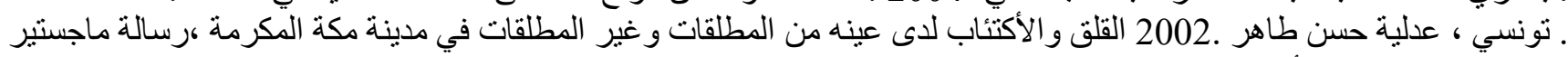

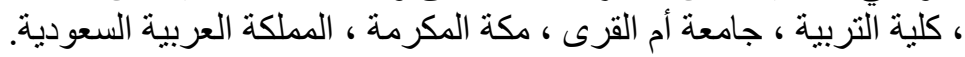

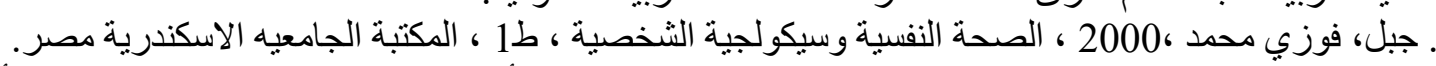

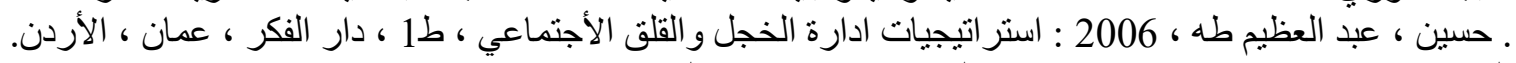

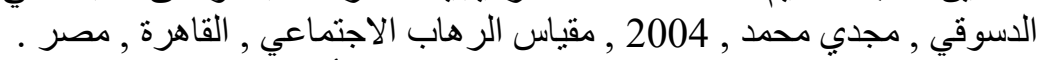

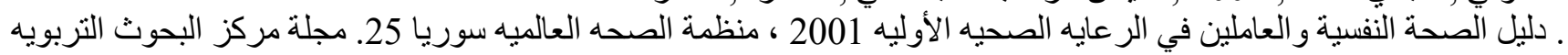

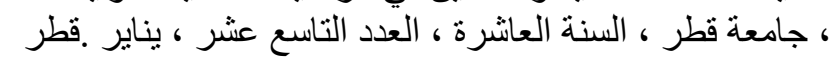

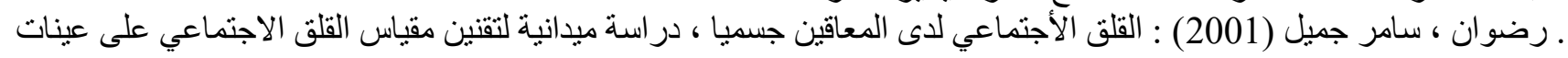
سورية. السبيعي , عبيد , 2006 , ان كنت خجو لا عالج نفسك بنفسك , وسائل علمية للتخلص من الخجل و الر هاب الاجنماعي ,مكتبة الملك فهر

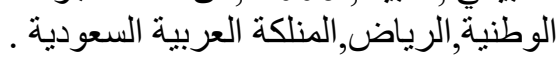

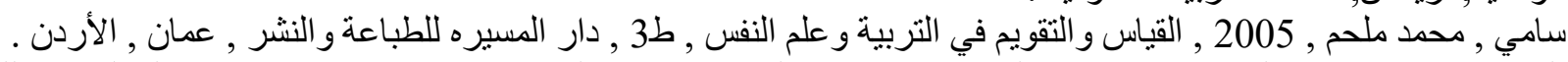
الثيخ , رو اء ناطق صالح , 2011, قياس الرهاب الاجتماعي لادى مستخدمي المحادثة عن طريق الانترنت , مجلة القادسية للعلوم

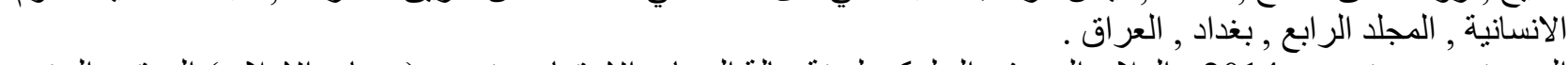

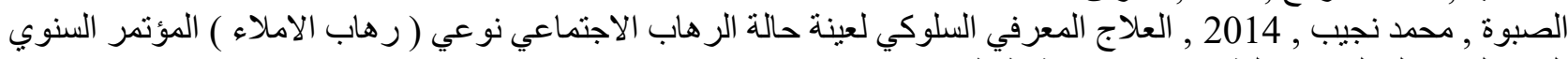

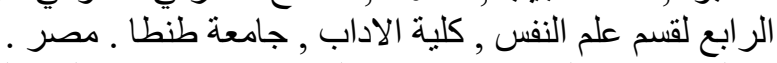

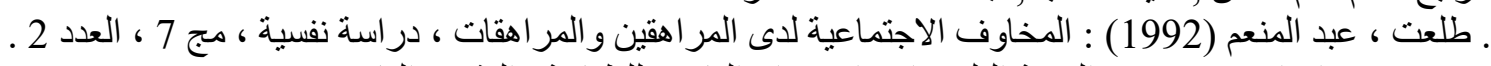

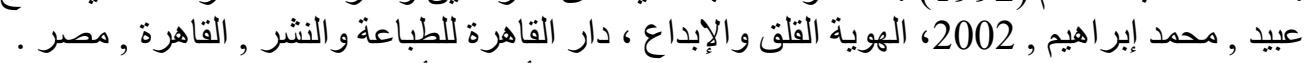

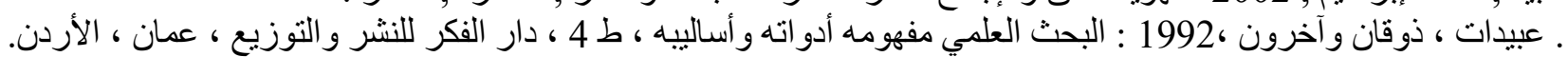

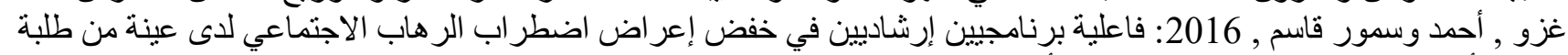

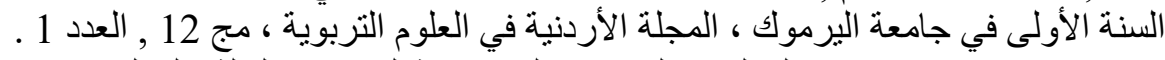

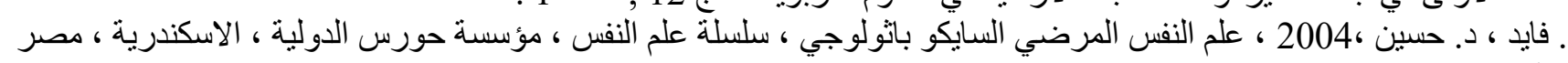
. في 1 b،

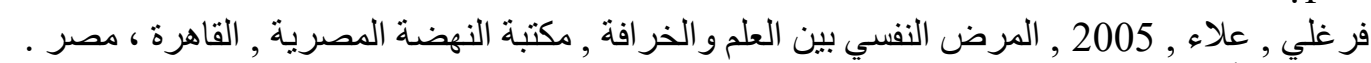

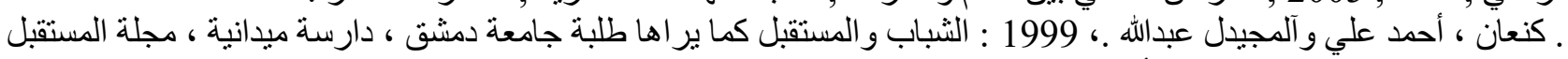

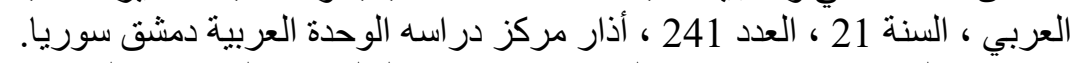
ـ ناصر ، المحارب ـ (2000) : العلاج الاستعر افي السلوكي ، الرياض ، السعودية السردية : دار الزهر اءه ، جامعة الزقازيق. 


\section{References}

Prince, Ali, (2002) Self-sufficiency, 1, House of Cultural Affairs, Iraq.

Amiri, Ahmed Ali Nahand, 2002: The Effectiveness of a Mentoring Program in Facing Mental Stress among High School Students, PhD Thesis, Faculty of Education, Mansoura University.

Abu Hneidi, d. Wael, 2003, social fear one of the diseases of the age, the network of informatics, No. 17, Zagazig University Egypt.

. Building, Adel al-Sayed and others 2006: Fear of oral communication and its relation to social anxiety and learning methods among a sample of English students in the Faculty of Education, unpublished doctorate, Future Journal of Arab Education, Modern University Office, Alexandria.

Bukhari, Mohamed Abdel Basset, Social phobia, 2004: An article on the site of the mental health hospital in Taif.

Tunisian, Adliya Hassan Taher. 2002 Anxiety and depression in the eyes of divorced and divorced women in the city of Mecca, Master Thesis, Faculty of Education, Umm Al Qura University, Makkah, Saudi Arabia.

Jabal, Fawzi Mohammed, 2000, Psychological and Psychological Health Personality, I 1, University Library of Alexandria, Egypt.

Hussein, Abdel-Azim Taha, 2006: Management Strategies for Shyness and Social Concern, 1, Dar AlFikr, Amman, Jordan.

Dessouki, Magdy Mohamed, (2004) Scale of social phobia, Cairo, Egypt.

. Guide to Mental Health and Primary Health Care Workers 2001, World Health Organization Syria

25. Journal of the Center for Educational Research, Qatar University, 10th Year, Issue 19, January.

Radwan, Samer Jamil (2001): Social Concern for Physically Disabled Persons, A Field Study to Regulate the Social Anxiety Scale on Syrian Samples.

Al-Subaie, Obaid, 2006, If you are shy, treat yourself yourself, scientific means to get rid of shame and social phobia, King Fahd National Library, Riyadh, Saudi Arabia.

Sami, Mohamed Melhem, 2005, Measurement and Evaluation in Education and Psychology, Vol. 3, Dar Al-Masirah for Printing and Publishing, Amman, Jordan.

Sheikh, Rawa Nateq Saleh, (2011) Measuring Social Phobia of Online Conversation Users, Qadisiyah Journal of Human Sciences, Vol. IV, Baghdad, Iraq.

Al-Sabwa, Mohamed Naguib,( 2014), Behavioral Cognitive Therapy for the Sample of the Case of Social Phobia (phobia of dictation) 4th Annual Conference of the Department of Psychology, Faculty of Arts, Tanta University. Egypt .

. Talaat, Abdel Moneim (1992) Social Concerns of Adolescents and Adolescents, Psychological Study, Vol. 7, No. 2.

Obaid, Mohamed Ibrahim,( 2002) Identity concern and creativity, Cairo Publishing House, Cairo, Egypt.

Obeidat, Toukan et al.,( 1992) Scientific Research, Its Concepts and Methods, 4, Dar Al Fikr Publishing and Distribution, Amman, Jordan.

Invasion, Ahmed and Samour Kassem, (2016): Effectiveness of two pilot programs in reducing the symptoms of social phobia disorder in a sample of first year students at Yarmouk University, Jordanian Journal of Educational Sciences, vol. 12, no. 1.

Fayed, d. Hussein, (2004) Pathology Psychologist, Psychological Series, Horus International, Alexandria, Egypt.

Farghaly, Alaa, (2005) Psychological Illness between Science and Myth, The Egyptian Renaissance Library, Cairo, Egypt.

. Kanaan, Ahmed Ali and Almajdil Abdullah, 1999: Youth and the Future as Seen by Damascus University Students, A Field Study, The Arab Future Magazine, Year 21, No.241, March. 
. Nasser, the warrior. (2000): Cognitive Behavioral Therapy, Riyadh, Saudi Arabia: Dar Al-Zahra, Zagazig University.

II. Foreign sources

-Baptista.C.A \& Loureiro.S.R,(2012), Journal (Journal of Affective Disorders) Volume (136) -Corkin,Y. \&Weinser,M. (2014) : The role of the college --- - classroom climate on acadmic procrastination ,learning and individual differences ,Vol ,32 ,p 294-303

-. Richards, Thomas, A. a social Anxiety Institute (ASl) (2004). Over coming social Anxiety Institute.

-.Pollack . -2004. The effectiveness of cognitive behavioral program collectively to address social anxiety disorder among young people.

-. Tiruwork, T.(2008): Academic Procrastination and causal perception of tabor senior secondary student Ethiopia African research review ,UMI Number 3287166. 